\title{
CLAROSCUROS EN LAS RELACIONES ENTRE LA ESCOLARIDAD Y EL TRABAJO. CONFIGURACIONES Y LÍMITES
}

\section{Lights and shadows in the relationships between schooling and work. Configurations and limits}

http://dx.doi.org/10.22235/pe.v9i2.1293

\author{
MARÍA DE IBARROLA*
}

Recepción: 7-06-2016

Revisión: 17-08-2016

Aceptación: 25-08-2016

Resumen: El artículo recupera la historia de la construcción de un cuerpo teórico que busca integrar el mayor número de elementos explicativos acerca de las relaciones entre la escolaridad y el trabajo. Se propone analizar el valor, para ello, de tres grandes categorías intermedias: una matriz de heterogeneidad estructural laboral, el concepto de proyecto socioeducativo de Estado (para la creación de escuelas orientadas a la formación para el trabajo) y el concepto de estructuras institucionales del currículum que prescriben la formación efectivamente ofrecida. El texto describe las aportaciones empíricas derivadas de cuatro grandes proyectos de investigación -y varias investigaciones puntuales- sobre esas relaciones, que han tomado como objeto de estudio poblaciones con distintos

\footnotetext{
* Maestra en Sociología (Universidad de Montreal) y doctora en Investigaciones Educativas (DIE Cinvestav). Desde 1977 es profesora-investigadora del Departamento de Investigaciones Educativas del Centro de Investigación y de Estudios Avanzados del IPN, México. Realiza investigaciones acerca de las políticas, las instituciones y los actores involucrados en las relaciones entre la educación y el trabajo, y las relaciona con temas de sociología de la educación, diseño curricular, política educativa en México, educación media y superior, y formación de investigadores.
} 
niveles de escolaridad, en diferentes sectores laborales, zonas geográficas de México y temporalidades.

Palabras clave: educación, trabajo, estructura heterogénea de producción, proyecto socioeducativo, estructuras institucionales del currículum

\begin{abstract}
The article recovers the history of the construction of a theoretical corpus that has tried to integrate a large number of explanatory elements about the relationship between schooling and work. The proposal is to analyze the value of three intermediate categories: a matrix of the heterogeneous nature of labor sectors in Mexico, the socio-educational projects for the creation of schools educating the labor force, and the curriculum institutional structures that mark the boundaries for an effective education. The text is developed through the description of four long standing research projects - and some other punctual research projects-, that have focused on populations with different levels of schooling in different labor sectors, temporalities and geographical zones in Mexico.
\end{abstract}

Keywords: education, work, heterogeneous labor sectors, socio educational projects, curriculum institutional structures

\title{
INTRODUCCIÓN
}

El artículo recupera la historia de la construcción de un cuerpo teórico que busca integrar el mayor número de elementos explicativos acerca de las relaciones entre la escolaridad y el trabajo. Se deriva de la realización consecutiva de cuatro grandes proyectos de investigación $-\mathrm{y}$ varias investigaciones puntuales - sobre esas relaciones, que han tomado como objeto de estudio a poblaciones con distintos niveles de escolaridad, en diferentes sectores laborales, zonas geográficas en México y temporalidades. ${ }^{1}$

\footnotetext{
${ }^{1}$ Estos proyectos han sido objeto de diferentes formas de presentación y tipos de publicación: ponencias en congresos, libros, capítulos de libro y artículos, que se refieren en la bibliografía final. El interés de esta presentación es la reflexión sobre los conceptos construidos y su pertinencia actual.
} 
Como parte del desarrollo de los proyectos se construyeron categorías intermedias para analizar cada uno de los polos de la relación: la heterogeneidad de la estructura laboral del país, para el polo del trabajo, y los proyectos socioeducativos centrados en la formación escolar para el trabajo y las estructuras institucionales del currículum, para el polo de la educación. De manera coyuntural, se han hecho acercamientos al significado diferenciado que tiene la escolaridad para conseguir trabajo e ingresos en una estructura laboral tan heterogénea, y al peso innegable de la formación escolar. Se identificó la diversidad de trayectorias posibles y tránsitos entre la escuela y el trabajo, incluso entre personas con la misma escolaridad, los conocimientos que ponen en juego los individuos al trabajar, más allá del conocimiento escolar y otras formas de aprendizaje y enseñanza de lo laboral. Los referentes escolares se situaron en el nivel medio y en el superior, en particular en el primero.

Más que preguntas de investigación, dos grandes inquietudes orientaron los proyectos:

1) Qué sucede en las relaciones entre la escolaridad y el trabajo ante el acceso a una mayor escolaridad, media o superior, por parte de grupos socioeconómicos que anteriormente no habían tenido esas oportunidades. Esto es, cuando se incrementa de manera explosiva el acceso de una población diversificada a una escolaridad cada vez mayor, más compleja y más segmentada, tal y como se ha dado en México a partir de la Reforma Educativa de 1970, que expandió la oferta pública y favoreció el crecimiento de la privada, en particular en los niveles superiores del sistema.

2) Cómo se decide la creación y desarrollo de instituciones escolares explícitamente orientadas a la formación para el trabajo, y qué elementos institucionales delimitan la eficacia de esas escuelas para la formación ofrecida a lo largo del tiempo. 
Las investigaciones se realizaron motivadas, en ocasiones, por inquietudes personales y, en otras, en respuesta a solicitudes puntuales del gobierno federal. Se desarrollaron conforme a diseños originales de los acercamientos teóricos y metodológicos que requerían las preguntas puntuales de cada gran proyecto, afianzados, sin duda, en la revisión de los avances de las teorías en su momento, y estuvieron mediadas por las oportunidades encontradas para el trabajo empírico. En dos ocasiones se recurrió a aproximaciones de tipo cuantitativo y estadístico; en dos más, a estudios cualitativos de caso. En todas las ocasiones hubo aproximaciones mixtas —análisis de documentos, análisis de estadísticas, encuestas, observaciones, entrevistas, visitas-, acompañadas de seminarios de estudio centrados en el seguimiento y reflexión sobre los avances.

Todos los proyectos fueron de gran envergadura, con una duración de cuatro a seis años ${ }^{2}$, en promedio uno por década. Algunos estudios puntuales se intercalaron en el tiempo, según su oportunidad. Para todos los proyectos se contó con el invaluable apoyo de los alumnos de maestría y doctorado en turno, quienes realizaron sus tesis de grado durante el desarrollo de estos, y que reciben los créditos correspondientes.

Se considera una ventaja inicial haber orientado la mirada a las relaciones desde lo escolar hacia lo laboral. En ese sentido, las investigaciones condujeron a una gran diversidad de espacios laborales empíricamente referidos por los egresados, lo que descartó restringir de antemano los problemas y las preguntas de investigación a las empresas modernas o las relaciones de clase en ellas, espacios privilegiados en esos momentos por los sociólogos del trabajo ${ }^{3}$.

A lo largo de casi 40 años se construyeron, revisaron y ampliaron algunas propuestas analíticas que resultaron especialmente propicias para explicar y delimitar los resultados encontrados, en el sentido de las teorías intermedias

\footnotetext{
${ }^{2}$ Se contempla desde el diseño inicial hasta la última publicación correspondiente, que no han podido contar con tiempo completo ni exclusivo.

${ }^{3}$ A principios del siglo XXI, se incorpora el estudio de los trabajos "atípicos" (De la Garza, 2000).
} 
(Merton, 1964; Buenfil, 2008): una matriz de heterogeneidad laboral, el concepto de proyecto socioeducativo del Estado y el concepto de estructuras institucionales del currículum, sobre cuya aportación y pertinencia actual interesa reflexionar.

\section{PRIMERA PARTE: UNA ESTRUCTURA LABORAL HETEROGÉNEA, DESIGUAL Y COMBINADA}

El crecimiento notable, en realidad explosivo, del acceso a la escolaridad superior en México a partir de la Reforma Educativa de 1970 (De Ibarrola, 1984) planteaba especificidades por explorar, más allá de la persistente presencia de mecanismos escolares y no escolares que favorecen correlaciones positivas entre los niveles socioeconómicos y la escolaridad alcanzada, o de enfocar la expansión de la escolaridad como intervención estatal para promover la acumulación de capital y la legitimación del sistema político. Las primeras fueron demostradas por la literatura de la época (Coleman, 1966; Baudelot y Establet, 1971; Bowles y Gintis, 1985; Bourdieu y Passeron, 1995) y han sido confirmadas hasta la fecha (Schwartzman, Cervini, Tedesco, Tenti, Llach, Rivero y De Ibarrola, 2005; Blanco Solís y Robles, 2014); la segunda ha sido descrita por diversos autores (Torres, 1989; 1995; Carnoy, Samoff, Burris, Johnston y Torres, 1990). Este tipo de resultados sugerían la reproducción continua de la sociedad, y el sistema de dominación capitalista y penetración de las desigualdades socioeconómicas más allá de la escolaridad. Ahora, sobre el ingreso al trabajo, ¿qué tanto ponían en duda el papel simplistamente atribuido a la escolaridad ${ }^{4}$ en la mejoría de ingresos y condiciones de trabajo?

Los investigadores, economistas y sociólogos motivados por la misma inquietud en otros países aportaban ya teorías que matizaban el rol propuesto por

\footnotetext{
${ }^{4}$ La teoría del capital humano no es de ninguna manera tan simplista como lo maneja el discurso político sobre la importancia de la escolaridad. Ya Becker (1983) incluía en su definición del capital humano la importancia de la capacitación y de la experiencia laboral. En las aproximaciones más recientes y reconocidas sobre el tema, Hanushek y Woessmann $(2012,2015)$ descartan el valor de los años de escolaridad cursados - en particular para América Latina - y demuestran correlaciones entre las competencias en matemáticas y ciencias, medidas por pruebas estandarizadas internacionales, y el desarrollo de los países.
} 
la teoría del capital humano. El argumento también era de tipo economicista: si crece la oferta de población económicamente activa (PEA) con escolaridad superior, disminuye su valor de cambio; lo que da lugar al subempleo ilustrado, la inflación, el credencialismo, el filtro, la teoría de la cola laboral y la competencia por los puestos de trabajo.

El enfoque educativo sobre las condicionantes socioeconómicas del acceso a una escolaridad mayor, y el planteamiento de la consecuente devaluación del valor de ese certificado, aportaban elementos teóricos relativamente familiares para delimitar los enfoques de investigación. En cambio, encararlos con una cierta ignorancia sobre las especificidades del trabajo al que se accede en función de la escolaridad requirió un esfuerzo muy intenso de estudio previo, que se tradujo en un largo seminario de análisis.

El detonador inicial del seminario fue cuestionar el imaginario colectivo según el cual un mercado de trabajo homogéneo opera con la misma racionalidad y los mismos mecanismos para toda la población demandante de trabajo y en todo tipo de empresas o emprendimientos laborales. Empíricamente, el contexto laboral inmediato de los investigadores, sus familias y conocidos ofrecía, a simple vista, un escenario muy complejo, compuesto por infinidad de datos sobre enormes diferencias y desigualdades en las condiciones de trabajo, tipos de ocupación, modos y cantidades de ingreso. Los referentes encontrados permitieron desmenuzar el significado de dichos datos mediante el análisis de un pequeño despacho de abogado (un abogado, una secretaria, dos pasantes) o un consultorio médico (médico, secretaria, enfermera) por contraste con lo que ocurre en las corporaciones de profesionistas ${ }^{5}$ que empezaban a perfilarse: cadenas de hospitales privados de propiedad nacional o internacional, o grandes firmas nacionales 0 internacionales de abogados, de contadores, de ingenieros, un destino laboral deseado por quienes alcanzaban la escolaridad superior. A simple vista era posible observar las oficinas de las grandes empresas internacionales,

\footnotetext{
${ }^{5}$ Mexicanismo que refiere a los trabajadores profesionales que tienen escolaridad superior.
} 
las cadenas de bancos y sus enormes rascacielos, que comparen el mismo espacio urbano con el comercio informal que invade las calles y las esquinas de la ciudad de México, las microempresas informales, los talleres domésticos, los vendedores ambulantes, los mil usos ${ }^{6} \ldots$

Estaban también al alcance del análisis, en un extremo, la formalidad de las plazas laborales en el sector público: estables, garantizadas de por vida, con una gran cantidad de prestaciones renegociadas año con año con el sindicato correspondiente; ganancias y privilegios laborales obtenidos por los sindicatos también en las grandes empresas privadas y, en el otro extremo, contrataciones temporales aun en el sector público, para obras muy puntuales, precarias, sin ninguna prestación, como las que afectan ahora a los miembros del nuevo "precariado" (Standing, 2013).

De entre todo ese caos de información, fue posible encontrar un orden basado en las teorías en boga sobre el trabajo. Diferentes autores (Altvater; Brooke; Carnoy; Carnoy y Carter; Gordon, Reich y Edwards; Hallack y Caillods; Muñoz Izquierdo; Singer, Tokman y Souza, citados por De Ibarrola y Reynaga, 1983) proponían ya la noción de estructuras laborales heterogéneas, enfoque que se completó con los planteamientos de la OIT sobre la economía y el trabajo informal, ${ }^{7}$ cuyas descripciones parecían muy semejantes a los trabajos callejeros observados. De la teoría marxista se recuperó la noción de la ganancia, acumulación de capital y, más que en la explotación de los trabajadores, el interés se centró en los logros y negociaciones sostenidos por las luchas sindicales y plasmados en la legislación laboral: las leyes, los contratos ley, los contratos colectivos de trabajo que registran la mayor formalidad posible en el país en las condiciones de contratación (Portes citado por De Ibarrola, 1988, p. 26).

\footnotetext{
${ }^{6}$ Mexicanismo que refiere a los trabajadores informales que se ofrecen para cualquier tipo de tarea remunerada.

${ }^{7}$ El informe de la OIT sobre Kenia, 1972 citado por Tokman (2003) Sorprende que las estadísticas laborales mexicanas no incorporan la categoría de trabajo informal sino hasta 2003

[http://www.inegi.org.mx/est/contenidos/proyectos/cn/informal/]. Apenas en 2010 proponen la matriz de Hussman (De Ibarrola Coord., 2014).
} 
La división y organización técnico-jerárquica del trabajo —en ocasiones muy compleja, en otras muy simple - fue una tercera gran consideración, pero no la menos importante: empresas que declaran más de 1000 posiciones laborales, mientras que otras solamente dos, patrones y trabajadores, o finalmente el simple "cuentapropista". Es una división estrechamente ligada con el desigual desarrollo tecnológico entre las empresas: desde la automatización, ya visible en algunas, hasta las tecnologías de cuarta o quinta generación, remendadas según la intervención imaginativa de los trabajadores, y también visibles en los pequeños talleres de todo tipo.

Entre tareas de análisis y de síntesis, se propuso la existencia de una estructura laboral heterogénea, desigual y combinada que, posteriormente, se calificó como una matriz de heterogeneidad laboral, basada en el rejuego de los indicadores y parámetros propios de esos tres grandes criterios, entre cuyos límites y rasgos se podrían delimitar —más adelante se privilegió el término configurar (De la Garza, 2000) - los espacios de análisis de las relaciones del tipo de trabajo con los alcances de la escolaridad lograda.

Los espacios laborales de la estructura heterogénea se delimitarían, entonces, en función de la interrelación de los parámetros propios de los tres grandes criterios que se identificaron:

\section{El sentido y orientación del trabajo, la racionalidad que lo guía}

- La acumulación de capital, la obtención de ganancia: empresas privadas, y algunas públicas, de propiedad nacional o internacional, clasificables en cualquier sector de la economía: agricultura, industria o servicios.

- La administración pública y la prestación de servicios públicos. Ejemplos claros eran el sistema educativo nacional o los sistemas de salud, vistos como espacios de trabajo y no solo organismos de gobierno. Posteriormente se justificó la incorporación de las organizaciones no 
gubernamentales como portadoras de esta racionalidad: de servicio público no gubernamental, no lucrativo (Girardo, 2003).

- La subsistencia del trabajador y su familia, aunque con diferentes modos y niveles de ingresos para esa subsistencia. Originalmente se pensó en los despachos de profesionistas en cierto extremo de bienestar y en los cuentapropistas de las esquinas en el otro extremo. Pronto fue posible adoptar el concepto antropológico de las unidades domésticas de producción (González de la Rocha citado por De Ibarrola, 1994), las micro y pequeñas empresas familiares, para finalmente identificar lo que uno de los estudiantes (Martínez, 2011) denominó la "racionalidad emocional", es decir, aquella que prioriza el bienestar de los miembros de la familia, lo que incluye la confianza de involucrarlos en los procesos laborales por encima de las ganancias posibles.

\section{El grado de formalidad/informalidad de las relaciones laborales}

Inicialmente se contempló este criterio como el apego o no a la ley, el cumplimiento o no de esta, aunque en muy diversos grados: contrato de trabajo, seguridad social, salarios mínimos, vacaciones, duración de las jornadas, prestaciones como aguinaldos, participación de utilidades. Pronto se hizo evidente la importancia de la institucionalidad en el acceso de empresarios y trabajadores a muy distintas facilidades institucionales (por ejemplo crédito, seguridad social, apoyos legales diversos). Este criterio se caracteriza por una diversidad de indicadores y grupos de indicadores: desde no tener literalmente ninguna condición de formalidad, como las trabajadoras domésticas o los comerciantes ambulantes, hasta tener bastante más prestaciones que las que marca la ley general, como los trabajadores del sector público sindicalizados o los cuadros dirigentes de las empresas trasnacionales.

En este aspecto se identificaron no solamente los múltiples rasgos de informalidad, sino también las distintas formas de interrelación de los trabajadores formales con los informales. Esto se hace visible en los comercios informales de 
alimentos en las esquinas de la ciudad de México, aprovechados por los empleados formales, o se descubre a partir de las cadenas de relaciones de proveeduría, comercialización, capacitación de la fuerza de trabajo, mecanismos informales de crédito o el subsidio por la vía de retrasos en la exigencia de los pagos entre los pequeños talleres informales y algunas empresas formales.

\section{La complejidad tecnológica y administrativa de las empresas}

Interesó no tanto el tamaño de la empresa en cuanto a su número de trabajadores, sino dos rubros, no necesariamente relacionados con él: la división técnico jerárquica del trabajo y el tipo de tecnología incorporada. Lo anterior permitía tener presente una doble aproximación, muy ligada con el conocimiento laboral y, en última instancia, con la escolaridad lograda: el nivel de dominio sobre el trabajo de otros y los niveles de conocimiento sobre la totalidad del trabajo productivo (De Ibarrola y Reynaga, 1983, p. 31):
a. Virtual $^{8}$
b. Automatizado
c. Semiautomatizado
d. Mecanizado
e. Artesanal
f. De autoría personal para subsistir

\footnotetext{
${ }^{8}$ Esta categoría se incorporó para un estudio del 2014.
} 
Figura 1: Matriz de heterogeneidad laboral

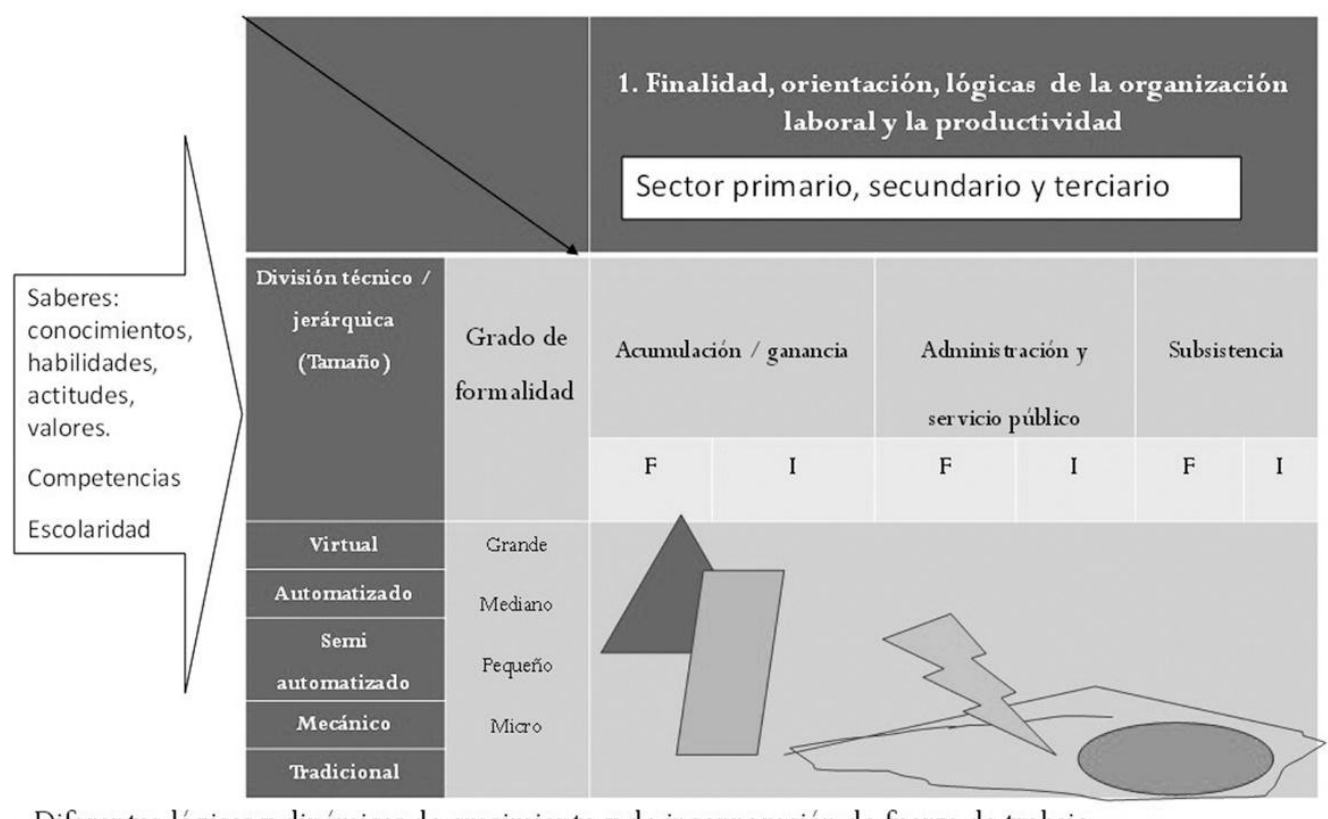

Diferentes lógicas y dinámicas de crecimiento y de incorporación de fuerza de trabajo

Fuente: De Ibarrola (Coord.), 2014.

Los espacios laborales derivados de la interacción entre los distintos parámetros de esos tres criterios (factorial $5 \times 3 \times 2$ ) no resultan especialmente precisos, ni estrictamente delimitados. Hay muchas interrelaciones que permitirían identificar espacios que ocupan una casilla o toda una línea o columna de la matriz, y no todos tendrían expresión real. Se localizaron, sí, ejemplos puntuales de la informalidad en los sectores más modernos y formales, como los niños empacadores dependientes exclusivamente de las propinas ${ }^{9}$ en las tiendas de autoservicio, los trabajadores sustitutos e interinos en el sector público o las condiciones laborales de informalidad y subsistencia en algunos de los últimos eslabones (talleres caseros) de la cadena de proveeduría a una gran empresa.

La importancia de esta matriz se incrementó al orientar la reflexión hacia el conocimiento de la relación entre sectores para la generación de empleos o la disponibilidad de qué tipo de trabajo, o la calidad y naturaleza de la modificación

\footnotetext{
${ }^{9}$ Posteriormente se ha reglamentado este trabajo.
} 
de los trabajos por desempeñar. Permitió cuestionar continuamente a las instituciones educativas sobre para qué trabajo formar (Jacinto, 2004).

Los cuatro grandes proyectos de investigación, que a continuación se describen, aprovecharon los principios de la matriz para delimitar y caracterizar la naturaleza de los espacios laborales en los que se situaba la población estudiada, enfocada de manera privilegiada en función de su escolaridad.

\section{Escolaridad superior y empleo formal (De Ibarrola y Reynaga, 1983)}

Un primer espacio laboral que fue posible investigar estaba situado entre los límites que marcan la acumulación de capital (empresas privadas de distintos giros, comerciales e industriales), relaciones formales de trabajo y una aguda división técnico-jerárquica de este. La oportunidad empírica la ofreció un grupo de 56 empresas privadas, que permitió el acceso a la información registrada para todas ellas por una misma oficina de análisis y valuación formal de puestos que las asesoraba sobre prestaciones y salarios competitivos. Se registró y analizó la escolaridad (en seis categorías, de primaria a posgrado), los ingresos (nueve categorías, desde 1 hasta 20 múltiplos del salario mínimo legal) y los puestos laborales (ocho categorías jerárquicas para ordenar 116 puestos diferentes) de 5968 empleados administrativos. Los resultados de este proyecto permitieron precisar:

- La preferencia, tanto de empleadores como de egresados de la educación superior, por este tipo de espacio: el $28 \%$ de los trabajadores contaban con ese nivel de escolaridad, porcentaje muy superior a la escolaridad de la PEA del país, que en esa época se situaba en un magro $3 \%$. 
- La significativa ${ }^{10}$ presencia de personal con este nivel de escolaridad junto con personal de menor escolaridad en ocho de las nueve categorías salariales y en siete de las ocho categorías jerárquicas.

- El desplazamiento de los mejores puestos de quienes solo tenían escolaridad superior por quienes contaban ya con posgrado, único nivel de escolaridad que aseguraba los ingresos y las posiciones más elevadas.

- La diferente y aún opuesta configuración de la distribución de los empleados administrativos según cada una de las variables estudiadas, lo que demuestra la difícil correlación entre ellas: la escolaridad, concentrada en la escolaridad media y superior; los ingresos, con una distribución agudamente piramidal; y la posición jerárquica, con una categoría central como la más frecuente y distribuciones inversamente piramidales hacia la posición superior y la inferior (De lbarrola y Reynaga, 1983, p. 67).

\section{Escuela y trabajo en el sector agropecuario: Los técnicos medios agropecuarios (De Ibarrola, 1994)}

Un segundo proyecto de investigación llevaría a conocer las relaciones de la escolaridad con el trabajo en el sector público. Se suponía la contratación formal, de conformidad con la ley Federal de Trabajadores al Servicio del Estado- para los egresados de los bachilleratos técnicos agropecuarios, en los programas gubernamentales de apoyo a la producción autosuficiente de alimentos (SAM) y a la productividad agrícola, en la posición jerárquica supuestamente necesaria del "técnico medio"11.

La oportunidad empírica la ofreció una solicitud del gobierno federal para evaluar las cooperativas de producción de estas escuelas. De ahí surgió un

\footnotetext{
${ }^{10}$ Una de las primeras sorpresas como investigadores nóveles fue no encontrar una correlación perfectamente lineal y positiva entre escolaridad, ingresos y jerarquía ocupacional.

${ }^{11}$ Como parte del estudio se hizo el rastreo del origen internacional del peso otorgado a esa posición laboral.
} 
proyecto adicional de investigación, que propuso un seguimiento puntual de todos los egresados de cuatro bachilleratos de la generación 1986-1988 en el contexto de cuatro mini regiones ${ }^{12}$ del país, que comparó sus aspiraciones laborales al momento del egreso con su realidad laboral un año después. Los datos arrojados por este proyecto permitieron dos grandes tipos de resultados, unos relacionados con el ingreso al trabajo de los egresados de estas escuelas y otros con la naturaleza de la formación escolar para el trabajo ofrecida por ellas.

Sobre el ingreso al trabajo de los egresados de estas escuelas:

- Se descubrió el cierre total de oportunidades laborales en el sector gubernamental para los egresados de la generación estudiada, debido a la suspensión de todos los programas públicos que previamente habían estado disponibles. La razón del cierre fueron las crisis financieras y el enflaquecimiento del Estado que afectó a muchos países de América Latina en la década de los ochenta. Fue un claro y extremo ejemplo de las diferentes racionalidades propuestas por María Antonia Gallart (1985), que se propusieron como lógicas contrarias entre los tiempos propios de la formación escolar y los tiempos propios de la apertura o cierre de oportunidades laborales. Anteriormente, los jóvenes habían sido reclutados por el sector público aún antes de concluir sus estudios; y luego, en los años en los que se hizo la investigación, ninguno de los jóvenes egresados logró empleo formal en el sector público.

- Se descubrió la creación de la categoría laboral de técnico medio como una iniciativa del sistema escolar. Una posición laboral existente conforme a la división del trabajo en el sector público, pero no en las empresas agroindustriales de las distintas zonas. Aunado al desconocimiento de la

\footnotetext{
${ }^{12}$ Un promedio de 20 estudiantes por plantel. Las mini regiones se delimitaron en función del alcance geográfico y las distancias entre las escuelas estudiadas y los domicilios de los alumnos. Se localizaron en cuatro entidades diferentes del país: Guerrero, Chiapas, Estado de México y Michoacán.
} 
posición en estas últimas, iba el desprecio por ese nivel de escolaridad (y por la juventud e inexperiencia de los jóvenes egresados). La preferencia de esos empresarios se inclinaba, si acaso, por algún experto de nivel superior y posgrado. Entre ellos lo que más funcionaba era la confianza en sus propios conocimientos empíricamente adquiridos.

- Se comprobó la dificultad de poner en juego el conocimiento adquirido en las escuelas para impulsar producciones agropecuarias, de manera personal o en las unidades familiares, por lo menos al año de haber egresado.

Estos tres resultados principales son ejemplo claro de las tensiones entre las lógicas educativa y laboral: los tiempos, las posiciones laborales y el tipo de conocimientos que entran en juego.

Sobre la naturaleza de la formación escolar lograda se acuñaron dos conceptos, el de proyecto socioeducativo del Estado ${ }^{13}$ (De Ibarrola, 1994, p. 26) y el de estructuras institucionales del currículum (Glazman y De Ibarrola, 1987; De Ibarrola, 2012). Ambos conceptos constituyen también herramientas teóricas que importa presentar en este texto y que se analizan más adelante, al referir al polo de la educación.

\section{Escuela, capacitación y aprendizaje en la industria del calzado (De Ibarrola, 2004)}

Para el tercer gran proyecto de investigación se contó con el apoyo de la Cámara de la principal industria de una ciudad de la zona centro de México, la del calzado. Se obtuvo una muestra de 36 empresas, que variaron desde empresas modernas y formales hasta talleres domésticos de producción (denominados, familiarmente, picas) y se entrevistó al total de sus trabajadores. Se obtuvo así una población de

\footnotetext{
${ }^{13}$ El concepto se anticipó en una reflexión publicada en 1988. Eventualmente, también permitió identificar otros proyectos socioeducativos, en particular los de empresarios privados o grupos religiosos al crear instituciones de educación superior.
} 
2200 trabajadores, muy diversa en cuanto a la naturaleza del centro de trabajo, la escolaridad alcanzada, los ingresos percibidos y las posiciones jerárquicas desempeñadas. En este proyecto se cambió el sentido que había orientado los dos proyectos anteriores: se partió del trabajo ocupado para preguntar cuál podría ser la relación con la escolaridad lograda, qué aprendizajes se ponían en juego y cómo y dónde se habían alcanzado dichos aprendizajes.

Los datos arrojados por el proyecto ofrecieron, además del tipo de relaciones entre escolaridad, ingresos y posición jerárquica, información fundamental sobre otros mecanismos para aprender a trabajar, que incluyeron:

- El hecho de que solamente la escolaridad superior definía diferencias en el acceso a mejores puestos y mayores ingresos.

- Otros niveles de escolaridad no resultaron el factor fundamental en la diferencia de ingresos, sino la edad (en contra de los más jóvenes) y el sexo (en contra de las mujeres).

- La importancia de la escolaridad técnica de nivel medio en ciertos espacios de la estructura laboral, en especial los espacios industriales.

- La inexistencia casi total de capacitación formal en las empresas (solo 6 de 36) y la prioridad que se da a la capacitación del personal de mayor escolaridad.

- Las empresas como espacios educativos importantes y el aprendizaje "silencioso" de los trabajadores (Mijares, 2003): un 86 \% declaró haber aprendido a trabajar en la fábrica, entre ellos, con algunos colegas como enseñantes, incluso el patrón mismo, y ellos como enseñantes de otros trabajadores. ${ }^{14}$

\footnotetext{
${ }^{14}$ Posteriormente fue posible interpretar estos datos con apoyo de la teoría del aprendizaje situado, como lo analizan Lave y Wenger (1991). Este descubrimiento orientó rápidamente a considerar la experiencia laboral - tan cara a los empresarios - como un concepto que, en forma empírica e intuitiva, da cuenta del aprendizaje situado.
} 
- Solamente $0.3 \%$ de los trabajadores con escolaridad primaria le dieron crédito a la escuela en su aprendizaje laboral. Quienes tenían escolaridad superior, pero en particular quienes contaban con una escolaridad técnica de nivel medio, señalaron en porcentajes muy superiores que habían aprendido a trabajar en la escuela: $27.6 \%$ y $25.6 \%$, respectivamente (De Ibarrola, 2004, p. 154).

- Aparece la evidencia del trabajo infantil en la dimensión de formación para el trabajo ${ }^{15}$ : prohibido por ley y limitado por esta prohibición, se manifestó inequívocamente presente en las empresas y en la vida de los trabajadores. Un $51 \%$ de los trabajadores reportaron haber realizado trabajo infantil: $77 \%$ de quienes desempeñaban tareas de supervisión, 13 $\%$ de quienes desempeñaban tareas administrativas, incluso $53 \%$ de quienes desempeñaban ya tareas de dirección (De lbarrola, 2004, pp. 148152).

- La formalidad de las empresas requiere importantes posiciones de gestión y administración, y es ahí donde es posible localizar el mayor número relativo de trabajadores con escolaridad media (administrativa) y superior.

\section{Claroscuros en las relaciones entre la educación y el trabajo}

El cuarto gran proyecto (2006-2015) en realidad fue la unificación de cuatro pequeños proyectos de tesis de doctorado, alrededor de la idea de que integrar el conocimiento sobre las relaciones entre educación y trabajo puede equipararse al armado de un enorme rompecabezas, sin piezas ni límites claros y algunas piezas se pueden delinear mejor con investigaciones cualitativas en profundidad.

El trabajo se constituyó por diversos acercamientos: a) entrevistas a 18 micro empresarios informales de la industria del vestido y observación en sus talleres (Martínez, 2011); b) observación continua de algunas esquinas de la

\footnotetext{
${ }^{15}$ En ningún momento se pretendió localizar o justificar el trabajo infantil, prohibido por la legislación, pero el dato fue evidente y notorio.
} 
ciudad y entrevistas con dos cuentapropistas (De Ibarrola, 2010); c) los estudiantes que trabajan y estudian a la vez (Cuevas de la Garza, 2013).

Estos acercamientos permitieron identificar la naturaleza de los "saberes productivos" que ponen en juego los trabajadores; las situaciones donde los adquieren y la manera de hacerlo; las relaciones entre distintos tipos de saberes: técnicos, de gestión, de relaciones; y las relaciones entre los saberes más sofisticados con una mayor escolaridad alcanzada ${ }^{16}$. Esto último se vio complementado por la innegable adquisición de competencias laborales entre los estudiantes que trabajan, lo que favorece su posterior incorporación a mejores trabajos (Cuevas de la Garza, 2013).

Un cuarto proyecto - basado en cuestionarios y entrevistas a todos los egresados de una misma carrera e institución- permitió identificar las muy diferentes transiciones que suceden de la Universidad al trabajo, a pesar de que los entrevistados contaran con igual escolaridad: misma institución, misma carrera, mismo período de formación (Valdivieso, s.f. $)^{17}$.

Dos estudios de interpretación de datos estadísticos censales aprovecharon las aproximaciones previas. Una base de datos elaborada por SITEAL ofreció información estadística sobre escolaridad e ingresos de la PEA mexicana, clasificada en cinco tipos de espacios laborales. Esto permitió identificar las brechas entre los ingresos de trabajadores con escolaridad superior y los de los trabajadores que no habían concluido la escolaridad primaria, en dos fechas diferentes.

Tabla 1: El valor de la educación superior en diferentes sectores laborales

\footnotetext{
${ }^{16}$ En el primer caso se llegaron a identificar las configuraciones diferentes de sistemas de negocios, los más formales relacionados con la mayor escolaridad, los menos formales con saberes muy reducidos.

${ }^{17}$ Una duda presente, por supuesto, tenía que ver con el origen socioeconómico de los estudiantes en esas diferentes transiciones, influencia que se percibió solo para las diferencias extremas.
} 


\begin{tabular}{|l|l|l|}
\hline Trabajadores en diferentes sectores & $\begin{array}{l}\text { Brecha de ingresos entre los que tienen } \\
\text { escolaridad superior y los que tienen } \\
\text { educación básica incompleta en cada } \\
\text { sector }\end{array}$ \\
\hline Ingresos promedio de la fuerza de trabajo & 1992 & 2004 \\
\hline Sector informal ( promedio) & 1 & 1 \\
\hline Trabajadores por su cuenta & 5.29 & 3.39 \\
\hline Trabajadores y microempresarios> 5 & .94 & 1.32 \\
\hline Sectores formales (promedio) & 4.95 & 3.33 \\
\hline Gobierno & 3.95 & 3.64 \\
\hline Industria & 4.18 & 3.48 \\
\hline Servicios & 6.82 & 3.71 \\
\hline Brecha promedio & 2.99 & 3.53 \\
\hline
\end{tabular}

Fuente: De Ibarrola, 2009a

Dos tipos de resultados llaman la atención: a) existe una brecha muy significativa entre los ingresos de los trabajadores con escolaridad superior y los que no completaron la escolaridad básica —los primeros reciben en promedio casi cuatro veces más-; b) entre las dos fechas analizadas la brecha disminuye, muy notablemente en el sector industrial, y solo se incrementa en dos sectores, el de la microempresa informal y el de los servicios.

Un tercer resultado no se aprecia en el cuadro: los datos puntuales sobre el monto de los ingresos permitieron afirmar que el personal con escolaridad superior siempre gana más que quienes tienen escolaridad básica incompleta al interior de cada sector, pero las diferencias entre trabajadores con la misma escolaridad superior entre sectores son importantes.

Un segundo proyecto puntual de investigación (De Ibarrola Coord., 2014), cuya finalidad fue identificar la problemática de los jóvenes en el ingreso al trabajo en la ciudad de México, permitió de manera colateral observar el panorama general de la distribución de la PEA conforme a los datos censales de 2010, según diferentes niveles de escolaridad y en cinco grandes categorías laborales: tasa de 
ocupación, tasa de desocupación, condiciones de informalidad y dos categorías de ingresos, los más elevados y los más precarios registrados.

Gráfico 1: Visión general de cinco rasgos laborales de la PEA de la Ciudad de México, por nivel de escolaridad

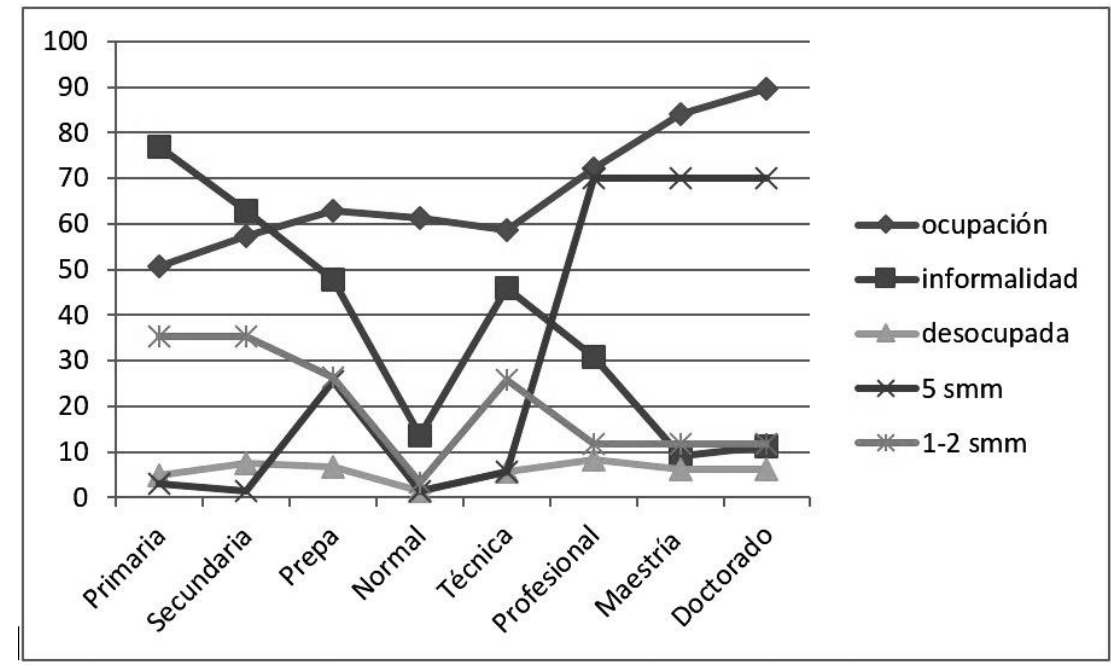

Fuente: De Ibarrola (Coord.), 2014.

El resultado que interesa destacar es la imagen estadística general en la que se observa al mismo tiempo la tendencia general a una correlación positiva entre las mejores condiciones en cada caso y la mayor escolaridad, pero también la presencia de población con todos los niveles de escolaridad en todas las condiciones, aun las más desfavorables. El desempleo, la informalidad y los bajos salarios, en particular el dato, tan preocupante actualmente, de la mayor participación de la PEA con mayor escolaridad en la tasa de desempleo. Una duda que no resuelven los datos estáticos presentados es si hubiera sido esperable esta distribución de la problemática entre quienes alcanzaron educación superior y posgrado décadas atrás o es efecto simple del crecimiento de la escolaridad. Una problemática semejante se observó en la primera investigación reseñada.

\section{SEGUNDA PARTE: LOS PROYECTOS SOCIOEDUCATIVOS PARA FORMAR ESCOLARMENTE PARA EL TRABAJO}


En el caso de México, han sido notorios el peso asignado al sistema escolar en la formación de la población para el trabajo y el concepto desarrollista, presente en todos planes y programas nacionales de educación y en la creación de cada una de las nuevas instituciones escolares de nivel medio y superior, a lo largo del período analizado, de 1970 a la fecha: los bachilleratos bivalentes, la formación profesional de nivel medio, la formación de profesionistas de quinto nivel, la importancia de las profesiones administrativas de nivel superior y el impulso decidido al crecimiento de las ingenierías.

La creación de instituciones escolares no se ha restringido a una propuesta exclusivamente economicista; otras funciones primordiales se han considerado en cada uno de los proyectos socioeducativos: la democratización del acceso y permanencia en la escuela, la equidad en el acceso al conocimiento y a la modernidad para todos los grupos sociales, la atención a la interculturalidad. La dimensión económica, sin embargo, ha sido la esencia de las expectativas puestas en las relaciones entre la escolaridad y trabajo, y de un vago e indefinido concepto de desarrollo, a pesar de todos los resultados que la matizan e incluso la contradicen.

\section{El proyecto socioeducativo del Estado ${ }^{18}$}

El concepto surge a raíz de la investigación sobre los técnicos medios agropecuarios, motivada por el interés de conocer los distintos ángulos de esta propuesta educativa conforme a un enfoque "lógico", que inició con las razones por las cuales se crearon los bachilleratos tecnológicos agropecuarios y concluyó con el estudio del destino laboral de sus egresados. Entre ambos puntos se hizo el análisis de la forma en que esos bachilleratos se distribuyeron a lo largo y ancho del país; la dotación material y humana que lograron los establecimientos escolares y el tipo de formación que se ofreció a la población asistente.

\footnotetext{
${ }^{18}$ De hecho, hubo un esfuerzo incipiente por identificar "proyectos socioeducativos de la burguesía".
} 
En cada uno de estos momentos de la propuesta surgió una complejidad de dimensiones: a) las razones políticas, económicas, gremiales y comunitarias para la creación esa oferta escolar: ofrecer oportunidades de escolaridad media superior a la población rural del país que ya rebasaba la escolaridad secundaria; formar el "técnico medio" que requería el desarrollo económico del sector; consolidar la formación escolar propia de ese nivel jerárquico en el ejercicio laboral de los programas de atención al medio rural; contener la demanda hacia la escolaridad superior; b) al analizar la creación misma de los establecimientos concretos en diferentes zonas del país, se descubrieron las diferencias en la capacidad de presión de los distintos actores: los gobernantes locales y los pobladores demandantes, y las razones en la negociación con el gobierno federal.

El proyecto socioeducativo se conceptualizó como la reconstrucción teórica que hace el investigador de la unidad de intención, racionalidad y fuerza de un conjunto de proposiciones y acciones (programáticas, legales, presupuestales, institucionales) del Estado mexicano para formar escolarmente -en este caso- a los técnicos medios agropecuarios que requerían sus programas de apoyo al campo como parte de un proyecto educativo y social más amplio. Al ser enfocada empíricamente esta reconstrucción, en el tiempo y en el espacio, se hacen patentes una serie de tensiones entre la unidad de enunciados y la diversidad de los procesos que involucran, o entre continuidades y cambios, intenciones y racionalidades, lagunas conceptuales y operativas, irracionalidades, acciones opuestas, vacíos... entre actores muy diversos (De Ibarrola, 1994, p. 26).

\section{Los actores en la creación de oportunidades escolares}

Una profundización posterior sobre el conjunto de actores, argumentos, razones y negociaciones evidentes en la construcción de los establecimientos escolares dedicados a la formación para el trabajo (De Ibarrola, 2014) permitió sistematizar la noción del rejuego entre cuatro diferentes tipos de actores.

Cabe señalar que en México las innovaciones escolares han surgido casi siempre por iniciativa del gobierno federal, cada vez más en convenio con 
gobiernos estatales y aun locales, que cuenta con distintos tipos de grupos técnicos especializados o los crea ex profeso, en ocasiones claramente influenciado por las políticas internacionales al respecto; en otras, como copias de instituciones creadas en otros países.

Un segundo actor, debidamente convocado o ciertamente tomado en consideración, aunque sea de manera indirecta (sujetos ausentes o de referencia), refiere a los representantes del sector laboral, casi siempre los empresarios organizados, en particular las cámaras nacionales, pero también las locales. La importancia de estos actores ha ido creciendo, ya que validan la pertinencia de la propuesta educativa respecto de las necesidades económicas del país en materia de formación para el trabajo. Por lo mismo, se han abierto diferentes maneras de participación en los organismos de planeación nacional o regional para la creación de establecimientos: en las juntas de gobierno, en los comités de diseño de planes, en la apertura de espacios para las prácticas y varias más.

Un tercer tipo de actor son los estudiantes y sus familias, la demanda: sus deseos y aspiraciones respecto de la escolaridad que quieren.

Finalmente, un cuarto tipo de actor son las instituciones escolares mismas, su historia y desarrollo, su orientación, sus objetivos, sus recursos.

Del estudio cualitativo sobre los bachilleratos agropecuarios surgió una gran variedad de elementos sobre la formación efectivamente adquirida por los jóvenes. Por un lado, la pertinencia de la propuesta educativa, esto es, la relación de los planes y programas con las supuestas necesidades del mercado de trabajo. Esta resultó claramente inadecuada en la fecha en que se realizó la investigación, precisamente porque se habían predefinido - como mercado de trabajo - las necesidades de los programas gubernamentales supuestamente orientados a la modernización de la agricultura, lo que se tradujo inicialmente en una enorme especialización de los programas ofrecidos (hasta 27 especialidades), con referencia a las grandes unidades capitalistas de producción y a la organización jerárquica de los organismos públicos orientados al sector. 
Por otro lado, su eficacia: ¿se había cumplido debidamente con la formación ofrecida? La dotación material y humana de los establecimientos escolares fue una de las dimensiones que más se analizaron en esa investigación. La información sobre los profesores que se contrataron, la velocidad a la que se hizo y la preparación de los contratados arrojó datos impresionantes sobre el reclutamiento de una gran cantidad de profesores sin la formación adecuada. Datos igualmente llamativos permitieron concluir que la dotación de equipamiento tecnológico había sido abrumadora para las primeras escuelas, y progresivamente deficitaria para las que se crearon posteriormente.

Además, la observación de los horarios y calendarios de estudio y los distintos espacios dedicados a ellos -las aulas, los talleres, los campos productivos - llevaron a la construcción de otro concepto que resultó sumamente útil para el conocimiento de la naturaleza y calidad de la formación que logran las escuelas: el de estructuras institucionales del currículum (Glazman y De Ibarrola, 1987; De Ibarrola, 2012).

Este concepto surge de la discusión en boga entre la eficacia de los planes de estudio — tal y como son diseñados, aprobados y legitimados por la autoridad facultada para ello- para conducir la formación de los jóvenes en tiempo y forma, y la "realidad curricular" (Jackson, 1968) puesta al descubierto por múltiples estudios etnográficos sobre la vida en las escuelas. ${ }^{19}$

El tono del debate orientaba a la posición de que las realidades curriculares resultan ajenas a lo que marcan los planes y los programas de estudios. El concepto propuesto identifica una serie de estructuras institucionales que ciertamente configuran, encauzan y eventualmente delimitan, y hasta determinan, la eficacia de la formación posible dentro de cada institución e incluso cada escuela: las planillas —normas - regulaciones, presupuesto disponible para la contratación de profesores; la asignación de tiempos y de espacios, los recursos didácticos disponibles; las formas de evaluación y en particular de certificación de

\footnotetext{
${ }^{19}$ María Antonia Gallart (2003) destaca la importancia de mirar las dinámicas institucionales en las escuelas técnicas y lo alejadas que están de lo que las políticas imaginan.
} 
los aprendizajes adquiridos que se justifican y legitiman precisamente por los planes de estudio debidamente autorizados (De Ibarrola, 1988, 2012).

En cada una de las escuelas visitadas durante esa investigación, las estructuras institucionales reales configuraban espacios muy limitados y deficientes para la formación "técnica" de los jóvenes. En cada establecimiento la consistencia entre las diferentes estructuras estaba rota u obstaculizada, empezando por la inadecuada formación de los profesores, el contrasentido de los tiempos y calendarios escolares, la ausencia de los espacios previstos para la producción conforme al modelo educativo, la ausencia de recursos materiales para ello, su descompostura o el desconocimiento de los maestros sobre su uso pedagógico ${ }^{20}$. La conclusión de un análisis que tome en cuenta las diferentes estructuras curriculares es sencilla: no se puede lograr un cambio o una mejoría si la innovación no integra los cambios correspondientes en cada estructura curricular y en su relación con las demás.

La posibilidad de estudiar la manera como estas estructuras se fueron construyendo a lo largo de la historia de una muy innovadora institución de educación media en el país (De lbarrola y Bazán, 1992) amplió los alcances del concepto, al poder explicar por qué ciertas estructuras curriculares no habían sido debidamente construidas en su momento, y la manera como limitaron y distorsionaron la eficacia de la formación ofrecida respecto de los objetivos buscados.

Otros proyectos puntuales posteriores permitieron ampliar la caracterización de las estructuras institucionales. Por ejemplo, la apertura de espacios educativos más allá de las escuelas, que se ha logrado con convenios de vinculación con las empresas cada vez más frecuentes, y la localización de nuevos actores formadores provenientes de las empresas (Durand, 2010) o la ampliación de los tiempos de estudio más allá de los burocráticos horarios escolares, con la

\footnotetext{
${ }^{20}$ Esta situación ha mejorado notoriamente. Esperamos que la investigación haya contribuido a ello.
} 
introducción de las comunicaciones electrónicas en los tiempos a voluntad de estudiantes y maestros.

Un descubrimiento muy importante fue la manera como el trabajo colegiado de un grupo de maestros - sin modificar formalmente el plan y los programas de estudios- logró una integración diferente de los contenidos de las asignaturas, sin cambiar tiempos y espacios formales, para impulsar las formas de trabajo necesarias para aplicar una educación basada en competencias (Mota Quintero, 2015).

\section{CONCLUSIÓN: RELACIONES ENTRE ESCOLARIDAD Y TRABAJO}

Los resultados reseñados fueron conformando, desde los primeros acercamientos a la problemática, una tesis general sobre la naturaleza de las relaciones entre la escolaridad y el trabajo (De Ibarrola, 1988). Se propuso que estas son complejas y plurales, no se pueden explicar solamente mediante un par de variables - los años de escolaridad y el nivel de ingresos - puesto que cada una de ellas está atravesada por diferentes desigualdades y constituida por rejuegos diferentes de múltiples actores. Estas relaciones son multidimensionales: no se dan aisladas o al margen de otras interacciones sociales; interactivas: se influyen la una a la otra; cambiantes en el tiempo y en el espacio: abarcan escalas espaciales y temporales de distinta magnitud; contradictorias: generan su propio agotamiento; históricas: influenciadas por su origen y su desarrollo en el tiempo, tanto en lo que refiere a las instituciones escolares como a las laborales; y aún perversas, en el sentido de lograr resultados opuestos a los propuestos.

Se rechazó el concepto de vinculación (en el sentido de supeditación) de las instituciones escolares al mercado de trabajo, pero se propuso la presencia de muy diversas formas y niveles de articulación entre ambos polos.

Se hizo evidente, a lo largo de los proyectos, que la escala a la que se manejan las relaciones modifica la evidencia a interpretar y orienta hacia diferentes políticas. A una escala censal macro-estadística, las correlaciones entre la escolaridad, el tipo de ocupación y los ingresos siguen siendo positivas, y 
apuntalan el interés, siempre presente, de lograr una escolaridad mayor. No son de ninguna manera exclusivas o absolutas, como lo señalan los datos sobre esas correlaciones en la ciudad de México y alimentan las desilusiones cada vez más frecuentes ante la mayor escolaridad.

En una escala media, las investigaciones permitieron precisar la intervención de muchas otras variables adicionales al simple dato de la escolaridad (en años) alcanzada por los trabajadores. Estas dependen de los sectores, ocupaciones, zonas geográficas y rasgos demográficos de la población como edad, género o condición de migración (De lbarrola, 2009b).

A escala individual aparecen todos los rasgos de la complejidad y la pluralidad de esas relaciones, mediante el análisis de las diferentes trayectorias (o transiciones), lineales y no lineales, que se dan entre la educación y el trabajo, y que afectan incluso a quienes provienen de una misma institución escolar y una misma carrera. Es posible profundizar entonces en aspectos como la naturaleza de la escolaridad lograda, e incluir el nivel, la especialización, la institución de origen, la capacitación formal recibida, la historia de la formación efectiva recibida en situación de trabajo y los cambios a lo largo de la vida del trabajador. Es ahí donde se marcan las contradicciones, 0 incluso las perversidades, entre la educación alcanzada y el trabajo logrado. Y describen también la importancia de la historia personal que puede construir cada individuo.

Resultó corroborada la tesis manejada por María Antonia Gallart, desde la década de los ochenta, sobre las racionalidades diferentes de lo educativo y lo productivo, que se reflejan en los momentos actuales con las teorías del adecuacionismo (Planas, 2014) o en las múltiples razones que propone Ulrich Teichler (2009) para las imperfecciones e inseguridades que impiden esa esperada funcionalidad de la escuela respecto del trabajo.

En cada polo de la relación se identificaron criterios determinantes para explicar esas limitaciones. Desde el punto de vista del trabajo, la heterogeneidad y la desigualdad de la estructura laboral y de las dinámicas de crecimiento definen la manera como la escolaridad juega en los diferentes espacios laborales, en 
diferentes contextos geográficos, sectoriales y en diferentes tiempos. Sin duda resulta necesario complementar el estudio de cada uno de esos espacios y la manera como las políticas del trabajo influyen en su crecimiento o transformación. Esa es una tarea que se ha realizado de manera muy parcial en las investigaciones reseñadas: la importancia que tuvo el cierre de los programas gubernamentales de apoyo al agro mexicano; un acercamiento histórico a la industria del calzado en la configuración de los distintos tipos de empresas que ahora la integran; ciertas precisiones sobre la naturaleza de la informalidad que afecta a las microempresas informales de la industria de la confección; descripciones empíricas sobre el trabajo en las esquinas (cruceros) de la gran ciudad.

El enfoque preferido por los proyectos ha sido la mirada desde lo educativo. Es ahí donde se han identificado con mayor intensidad rubros que describen la complejidad y la pluralidad de lo que pasa en las escuelas que forman para el trabajo: desde la decisión de crearlas y las determinaciones puntuales que imponen los actores que participan en ello, hasta la configuración concreta, histórica, de las estructuras institucionales curriculares que delimitarán su operación cotidiana (y la calidad de la formación que ofrecen) así como su impacto social, observado a través del seguimiento puntual de los egresados.

La estrategia de llevar en paralelo un seminario de estudios sobre el trabajo, conservada a lo largo de todos los proyectos de investigación, ha permitido ir revisando las diferentes categorías intermedias que se propusieron, generar adaptaciones, cambios e introducir nuevos elementos.

La matriz de heterogeneidad laboral fue muy útil para conformar una visión de conjunto sobre las diferentes lógicas o racionalidades que guían la creación del trabajo, la existencia tan numerosa de trabajos informales que difícilmente se explican como trabajo atípico, así como la coexistencia en el tiempo y el espacio de diferentes tecnologías y la división del trabajo que sustentan. Fue útil para cuestionar los objetivos buscados en la creación de nuevas instituciones escolares 
y sustentar las críticas a las indiscriminadas ofertas de mejorar el empleo y los ingresos, que las han acompañado.

El concepto de proyecto socioeducativo orientó a identificar el enfrentamiento entre múltiples y desiguales actores, sus intereses, argumentos y capacidad de presión sobre la racionalidad de la formación a ofrecer, que determinan la naturaleza del resultado logrado.

El concepto de estructuras institucionales del currículum fue muy útil para argumentar una de las causas más importantes del fracaso o ineficiencia de innovaciones o reformas curriculares, con la simple evidencia de que no se atiende la importancia de cada una de las estructuras institucionales, según su naturaleza, y la necesaria interrelación entre ellas.

A estas alturas parece indispensable revisar el valor explicativo de los tres conceptos. Con relación a la matriz de heterogeneidad estructural, se perfilan ya una serie de nuevos trabajos que solo de manera muy forzada se colocan en el tipo de espacio que configuran los tres criterios básicos que propone la matriz. Cómo describir los "nuevos yacimientos de empleo"; dónde colocar a los profesionistas autónomos de la nueva generación; cómo categorizar la "economía del cuidado" o el ímpetu de las tecnologías en la transformación de las condiciones y organización de las relaciones de trabajo para todos los trabajos "virtuales"; dónde clasificar la uberización $n^{21}$ de las relaciones laborales o el espacio que ocupa; hasta qué punto el criterio de informalidad explica el trabajo "flexible", o la precariedad laboral se explica por los mismos indicadores de la informalidad; qué referencias dan estos nuevos trabajos y relaciones laborales a las instituciones de formación técnica y profesional.

Son evidentes los profundos cambios en el tipo de formación que requieren todas estas transformaciones laborales, que exigen una nueva concepción de trabajo y una formación sistemática e integral a lo largo de la vida. Se trataría de

\footnotetext{
${ }^{21} 11550$ referencias aparecieron al consultar el término en Google, el 20 de junio de 2016. La economía colaborativa, el consumo colaborativo (https://www.enriquedans.com/2014/12/la-uberizacion-comoconcepto.html).
} 
proyectos socioeducativos diferentes en los que distintos actores respondan al reto de la heterogeneidad, pero, en particular, que enfrenten la desigualdad que ha conllevado y ante cuyas magnitudes la propuesta dominante actual de formar por competencias queda sumamente corta. Los cambios afectan también seriamente a las instituciones escolares: a las estructuras curriculares mismas, la profesionalidad de los profesores, los usos del tiempo, de los espacios, los recursos virtuales disponibles, formas de evaluar y certificar que están cambiando drásticamente por la educación a distancia, la educación virtual (y las prácticas laborales virtuales), así como el reconocimiento de una necesaria articulación en tiempo y forma entre la experiencia escolar y experiencia laboral.

Se trata, como se propuso en un artículo muy reciente (De Ibarrola, 2014), de identificar la nueva naturaleza de las "relaciones flojamente acopladas" que se dan entre la educación y el trabajo, ante los cambios drásticos que sufre cada uno de esos polos y entre sí, porque algunas de sus variables coinciden —no todas, ni siempre las mismas-, pero ciertamente la educación y el trabajo tienen importantísimos puntos de contacto que ofrecen desafíos muy inquietantes a la investigación y a la política educativa.

\section{REFERENCIAS:}

Baudelot, C. y Establet, R. (1971). La escuela capitalista en Francia. España: Siglo $\mathrm{XXI}$.

Becker, G. S. (1983). El capital humano: Un análisis teórico y empírico referido fundamentalmente a la educación. Madrid: Alianza.

Blanco, E., Solís, P. y Robles, H. (2014). Caminos desiguales. Trayectorias educativas y laborales de los jóvenes de la ciudad de México. México: INEE/Comex.

Bourdieu, P. y Passeron, J. C. (1995). La reproducción. México: Fontamara.

Bowles, S. y Gintis, H. (1985) La instrucción escolar en la América capitalista. Madrid: Siglo XXI. 
Buenfil, R. N. (2008). La categoría intermedia. Cruz Pineda, O. y Echavarría Canto, L. (Coords.). Investigación social: Herramientas teóricas y Análisis Político de Discurso (pp. 29-40). México: Juan Pablos.

Carnoy, M., Samoff, J., Burris, M. A., Johnson, A., Torres, C.A. (1990). Education and Social Transition in the Third World. New Jersey: Princeton University Press.

Cuevas de la Garza, J. F. (2013). La figura del estudiante-trabajador en el contexto de las relaciones entre educación escolar y empleo. [Tesis doctoral], Cinvestav-IPN, México.

De Ibarrola, M. y Reynaga, S. (1983). Estructura de producción mercado de trabajo y escolaridad en México. Revista Latinoamericana de Estudios Educativos, 8 (3), 11-81.

De Ibarrola, M. (1984). El crecimiento de la escolaridad superior en México como expresión de los proyectos socioeducativos del Estado y la burguesía. Revista Mexicana de Sociología, XLVI (2), 173-244.

De Ibarrola, M. (1988). Hacia una reconceptualización de las relaciones entre el mundo de la educación y el mundo del trabajo en América Latina. Revista Latinoamericana de Estudios Educativos, 2, 9-64.

De Ibarrola, M. y Bazán, J. (1992). La construcción institucional del currículum en la historia del $\mathrm{CCH}$. $\mathrm{CCH}, 6,9-16$.

De Ibarrola, M. (1994). Escuela y trabajo en el sector agropecuario en México. México: Miguel Angel Porrúa/ Cinvestav/ Instituto Mora/ Flacso.

De Ibarrola, M. (Coord.). (2004). Escuela, capacitación y aprendizaje. La formación para el trabajo en una ciudad en transición. Montevideo: CINTERFOR/OIT.

De Ibarrola, M. (2009a). Priorité à la formation scolaire pour le travail au Mexique. Tensions et contradictions entre l'Etat, les secteurs professionnels et les étudiants. Formation Emploi, revue française de sciences sociales, 107, 2539.

De Ibarrola, M. (2009b). El incremento de la escolaridad de la PEA en México y los efectos sobre su situación aboral y sus ingresos, 1992-2004. Revista 
Electrónica de Investigación Educativa, 11 (2), 1-19. Recuperado de: http://redie.uabc.mx/redie/article/view/232

De Ibarrola, M. (2009c). Siete preguntas clave sobre las relaciones entre la educación y el trabajo. ¿Qué respuestas aporta la investigación educativa? De Alba, A. y Glazman, R. (Coords.) ¿Qué dice la investigación educativa? (pp. 315-368). México, D.F.: COMIE.

De Ibarrola, M. (2010). Knowledge for a street corner economy. Presentado en la Conferencia anual de la American Education Research Association. Denver, Colorado.

De Ibarrola, M. (2012). Experiencias y reflexiones sobre el diseño y la evaluación curricular. Landesmann Segall, M. El currículum en la globalización. A tres décadas de el currículum pensado y el currículum vivido (pp. 93-119). México: UNAM/ FES.

De lbarrola, M. (2014). Repensando las relaciones entre la educación y el trabajo: una reflexión basada investigaciones realizadas en México. Cadernos CEDES, 34 (94), 367-383.

De Ibarrola, M. (Coord.). (2014). La formación para el trabajo en la ciudad de México. Informe sobre la problemática de la transición del sistema educativo al mercado de trabajo y las políticas públicas para enfrentarla en los últimos 17 años. México: Cinvestav/Secretaría de Educación.

De la Garza, E. (Coord). (2000). Tratado latinoamericano de sociologia del trabajo. México: El Colegio de México/ Facultad Latinoamericana de Ciencias Sociales/ Universidad Autónoma Metropolitana/ Fondo de Cultura Económica.

Durand Allison, M. C. (2010). Vinculación entre educación y trabajo. Los espacios formativos para el trabajo en una escuela Conalep. [Tesis de maestría]. Cinvestav-IPN, México.

Gallart, M. A. (1985). La racionalidad educativa y la racionalidad productiva: las escuelas técnicas y el mundo del trabajo. Buenos Aires: Centro de Estudios de Población. 
Gallart, M. A., Miranda, M., Peirano, C. y Sevilla, M.P. (2003). Tendencias de la Educación Técnica en América Latina, Estudios de Caso en Argentina y Chile. París: IIPE-UNESCO.

Girardo, C. (2003). La formación de los jóvenes para el trabajo desde las organizaciones de la sociedad civil. Desafíos y estrategias. [Tesis doctoral]. Facultad de Ciencias Políticas y Sociales de la Universidad Nacional Autónoma de México, México.

Glazman, R. y De Ibarrola, M. (1987). Planes de estudios. Propuestas institucionales y realidad curricular. México: Nueva Imagen.

Hanushek, E. A. y Woessmann, L. (2012). Schooling, Cognitive Skills, and the Latin American Growth Puzzle. Journal of Development Economics, 99 (2), 497-512.

Hanushek, E. A. y Woessmann, L. (2015). Universal basic skills. What countries stand to gain. OECD Publishing. http://dx.doi.org/10.1787/9789264234833en

Jacinto, C. (Coord.). (2004). ¿Educar para qué trabajo? Discutiendo rumbos en América Latina. Buenos Aires: redEtis (IIPE-IDES)/MTEySS/MECyT/La Crujía.

Jackson, P. W. (1968). La vida en las aulas. Madrid: Morata.

Lave, J. y Wenger, E. (1991). Situated Learning. Legitimate peripheral participation. New York: Cambridge University Press.

Martínez López, E. A. (2011). Los saberes productivos: sus configuraciones y usos en el caso de la microempresa de la confección. [Tesis doctoral]' Cinvestav-IPN, México.

Mijares Ruiz, Al. (2003) Aprendizajes silenciosos de los jóvenes trabajadores en la ciudad de León. [Tesis de maestría]. Universidad Iberoamericana de León/Cinvestav, México.

Merton, R. K. (1964). Teoría y estructura sociales. México: FCE. 
Mota Quintero, M. A. (2015). Las universidades tecnológicas de desarrollo: un caso de formación laboral por competencias. [Tesis doctoral]. CinvestavIPN, México.

Planas, J. (2014). Adecuar la oferta de educación a la demanda de trabajo. ¿Es posible? Una crítica a los análisis "adecuacionistas" de relación entre formación y empleo. México: ANUIES.

Schwartzman, S., Cervini, R., Tenti, E., Llach, J.J., Rivero, J., Tedesco, J. C. y De Ibarrola, M. (2005). Equidad en el acceso y la permanencia en el sistema educativo. SITEAL. Serie debates, 1.

Standing, G. (2013). El precariado. Barcelona: Pasado y presente

Teichler, U. (2009). Higher Education and the World of Work: Conceptual Frameworks, Comparative Perspectives, Empirical Findings. Rotterdam: SensePublishers.

Tokman, V. (2003). De la informalidad a la modernidad. Boletín Técnico Interamericano de Formación Profesional, 155, 9-32.

Torres, C. A. (1989). The Capitalist State and Public Policy Formation: A Framework for a Political Sociology of Educational Policy-Making. The British Journal of Sociology of Education, 10 (1), 81-102.

Torres, C. A. (1995). Teorías de la expansión educativa y la masificación escolar: para pensar la universidad pública en América Latina. Perfiles Educativos, 69, 7-20.

Valdivieso Martínez, Azul (s.f.) Trayectorias laborales de los egresados de las carreras de Ciencias de la Educación y Ciencias de la Comunicación de la Universidad Autónoma del Estado de Hidalgo. [Tesis doctoral en proceso] Cinvestav-IPN, México. 